disposición. Si se toma en cuenta que el aborto con medicamentos no es necesariamente el método adecuado para todas las mujeres, se debe evitar que el costo sea el factor decisivo en la elección. El apoyo psicosocial que las mujeres reciben durante el proceso de aborto resulta crucial, especialmente si consideran que el aborto es un pecado, son jóvenes o pobres, no conocen bien su organismo, carecen del apoyo de su pareja o quedaron embarazadas como resultado de actos de violencia sexual. (Lafaurie MM et al. Women's perspectives on medical abortion in Mexico, Colombia, Ecuador and Peru: a qualitative study. Reprod Health Matters. 2005;13(26):75-83.)

\section{Abandono espontáneo del hábito de fumar durante el embarazo en mujeres estadounidenses de bajos ingresos}

Diversas investigaciones han asociado el hábito de fumar cigarrillos durante el embarazo con el peso bajo al nacer, el nacimiento prematuro, el aumento del riesgo de complicaciones durante el parto y el aborto espontáneo. Por ello es importante conocer los factores que favorecen que una mujer abandone de manera espontánea ese hábito y saber si ese abandono será prolongado.

En este estudio preliminar se comparan algunas características de las mujeres de bajos ingresos que abandonaron espontáneamente el hábito de fumar cigarrillos con las de mujeres que mantuvieron ese hábito. Participaron en él 141 mujeres embarazadas que fumaban cigarrillos o que habían abandonado ese hábito sin ayuda profesional al saber de su embarazo. Las participantes eran mayores de 18 años y tenían menos de 30 semanas de gestación. Las mujeres que abandonaron el hábito de fumar espontáneamente se dividieron aleatoriamente en dos grupos: uno recibió tratamiento especializado para evitar que se volviera a fumar y otro, el grupo testigo, recibió el tratamiento habitual.

En total, 33 mujeres (23\%) abandonaron espontáneamente el hábito de fumar cigarrillos. Los resultados demostraron que estas mujeres tenían más confianza en sí mismas, eran más jóvenes y fumaban menos que las que no dejaron de fumar. Cada una de estas variables tuvo una contribución independiente de $25 \%$ al abandono espontáneo del hábito de fumar.

Al final del período de gestación, $76 \%$ de las participantes habían dejado de fumar y $36 \%$ del total seguía sin fumar 6 meses después del parto. La psicoterapia no aportó ninguna protección adicional contra la recaída en el subgrupo que dejó de fumar espontáneamente.

Los datos derivados de esta investigación, junto con otros obtenidos en investigaciones anteriores, indican que si bien es baja la tasa de recaída inmediatamente después del parto en las mujeres que abandonaron espontáneamente el hábito de fumar cigarrillos, la mayoría de estas habían retomado ese hábito 6 meses después. Se deben reforzar las acciones dirigidas a evitar la recaída en el período posterior al parto.

Estos resultados se basan en entrevistas con mujeres que aceptaron participar voluntariamente en el estudio, del que formaba parte una intervención para dejar de fumar, por lo que no son representativos de todas las mujeres embarazadas que fuman. No obstante, dada la importancia que tiene para la salud pública el abandono de este hábito nocivo, especialmente en mujeres embarazadas, estos resultados pueden ayudar a los proveedores de salud a estructurar campañas antitabáquicas más eficaces dirigidas a este grupo de la población. (Morasco BJ et al. Spontaneous smoking cessation during pregnancy among ethnic minority women: a preliminary investigation. Addict Behav. 2006;31(2):203-10.)

\section{Riesgo de transmisión de la tripanosomiasis americana por triatominos en México}

La tripanosomiasis americana, una de las principales enfermedades transmitidas por insectos en América Latina, es endémica en varios estados de México. En ese país la seroprevalencia es de 1,5\% en donantes de sangre y asciende a $20 \%$ en la población de algunas regiones. Además, en los últimos años se han diagnosticado varios casos de la enfermedad.

En México se conocen 31 especies de triatominos que pueden ser vectores del agente causal de la enfermedad y 18 de ellos están infectados de forma natural con el parásito Trypanosoma cruzi. La mayoría de las especies encontradas en las viviendas y en sus alrededores son de origen selvático.

Para caracterizar el nivel de infestación doméstica y peridoméstica con triatominos se estudiaron tres comunidades rurales de México: Felipe Carrillo Puerto (Estado de Nayarit), Cuxpala (Zacatecas) y Tepehuaje de Morelos (Jalisco) entre abril de 1998 y noviembre de 1999. Estas comunidades están ubicadas en regiones semiáridas, tienen condiciones atmosféricas similares y nunca han for- 
mado parte de programas de control antivectorial. La gran mayoría de las viviendas están construidas con materiales modernos.

Se encontraron índices muy elevados de infestación (entre 26,1\% y 58,3\%) y de colonización (de $58,3 \%$ a $85,7 \%$ ) alrededor de las viviendas. Solamente se constató la infestación dentro de las viviendas en la comunidad de Cuxpala con Triatoma longipennis. Un estudio preliminar reflejó la tendencia al aumento de la población de triatominos y de los índices de infestación durante la temporada seca. La tasa de infección de los triatominos fue de $41,2 \%$ a $60,2 \%$ y todos los flagelados encontrados se identificaron como Trypanosoma cruzi.

Algunas especies del complejo de T. phyllosoma se encontraron con frecuencia en Tepehuaje de Morelos y Felipe Carrillo Puerto; fueron especialmente abundantes las especies T. longipennis, T. pallidipennis y T. picturata. En este último sitio se obtuvieron ejemplares de T. longipennis y T. picturata; no se observaron formas híbridas a pesar de las condiciones favorables para la hibridación natural.

En Tepehuaje de Morelos, T. longipennis fue la especie más frecuente; estaba ampliamente establecida en las áreas peridomésticas, donde formaba colonias, como lo demostraron el elevado índice de infestación y la alta proporción de ninfas en las muestras recolectadas. En Cuxpala, T. longipennis fue el único vector detectado, aunque en comparación con Tepehuaje de Morelos, el índice de infestación fue menor, se observaron menos colonias y la infestación se extendió tanto por los alrededores de las viviendas como en su interior. Los índices de colonización y de ninfas en esa ciudad fueron más bajos que en las otras localidades estudiadas.

En Felipe Carrillo Puerto, las características epidemiológicas fueron muy similares a las encontradas en Tepehuaje de Morelos, con un índice de infestación más bajo; la especie principal en la zona fue $T$. picturata.

El nivel actual de infestación con triatominos y la elevada tasa de infección de estos con Trypanosoma cruzi constituyen factores de riesgo de transmisión de la tripanosomiasis americana. Las mayores tasas de infestación observadas durante la temporada seca pueden estar asociadas con un incremento de la actividad de los insectos y con un aumento de su capacidad para invadir nuevos sitios cuando la temperatura es mayor. Se deben emprender estudios de seroprevalencia que permitan evaluar la situación epidemiológica en esta zona. (Magallón-Gastélum E et al. Epidemiological risk for Trypanosoma cruzi transmission by species of Phyllosoma complex in the occidental part of Mexico. Acta Trop. 2006;97:331-8.)

\section{La temporada de picadura de Anopheles darlingi y su relación con el control del paludismo en Bolivia}

El paludismo en Bolivia tuvo un resurgimiento en los años noventa, particularmente en la parte amazónica del norte del país que colinda con el Brasil, donde su frecuencia aumentó cuatro veces entre 1991 y 1998. El aumento se debió en gran parte a que en ese territorio la deforestación llevó a un mayor contacto entre el mosquito vector y nuevos pobladores que carecían de inmunidad. La mayor parte de los casos de paludismo en la Amazonía boliviana se deben a la infección por Plasmodium vivax, aunque $P$. falciparum abunda también. Son varios los vectores que se encuentran en el territorio, siendo el principal de ellos Anopheles darlingi Root. Se trata de un vector muy eficaz debido a su intensa antropofilia y relativa longevidad. Los hábitos de picadura de este mosquito se conocen poco y fueron el objeto de un estudio realizado durante la temporada seca en julio de 2003 en El Prado, distrito del pueblo de Riberalta en la provincia de Beni, en la Amazonía boliviana.

Dos entomólogos voluntarios dotados de antorchas se sentaron al aire libre en una zona a 10 metros de distancia de la vivienda más cercana donde se habían producido picaduras de mosquito, según informes. Atraparon a todos los mosquitos que se les posaron en el cuerpo entre las $18.00 \mathrm{y}$ las $6.00 \mathrm{~h}$ (desde media hora antes del anochecer hasta media hora después del amanecer), tomando nota del momento exacto en que cada mosquito se posó. Los mosquitos fueron atrapados, transportados a un laboratorio en recipientes especiales y sometidos a análisis para la identificación de la especie.

Se atraparon vectores anofelinos de las siguientes especies: A. darlingi (272), A. albitarsis s.l. (16) y $A$. braziliensis. Cuarenta y ocho por ciento de las picaduras se produjeron entre las $19.00 \mathrm{y}$ las 21.00 h y $83 \%$ de todas las picaduras habían ocurrido antes de las $22.00 \mathrm{~h}$, que es cuando la mayoría de las personas se van a dormir.

Los hábitos de picadura de los anofelinos se ven afectados por la temperatura y la densidad poblacional. El estudio se realizó al aire libre en la temporada seca, y es posible que en el interior de las 